\title{
Radio Resource Management Approaches for Reliable Device-to-Device (D2D) Communication in Wireless Industrial Applications
}

\author{
Idayat O. Sanusi ${ }^{1}$, Karim M. Nasr ${ }^{1,2}$ and Klaus Moessner ${ }^{2}$ \\ 1. Faculty of Engineering and Science, University of Greenwich, Kent, ME4 4TB, United Kingdom \\ 2. Institute for Communication Systems, 5G IC, University of Surrey, Guildford, GU2 7XH, United Kingdom \\ \{i.o.sanusi, k.m.nasr\}@gre.ac.uk, \{k.nasr, k.moessner\}@surrey.ac.uk
}

\begin{abstract}
The manufacturing industry is regarded as one of the most demanding verticals with respect to URLLC requirements. A Device-to-Device (D2D) communication system is a key enabler that has been introduced to support URLLC. To enhance spectrum utilisation, D2D links can share access of radio spectrum resources occupied by cellular users. Spectrum reuse may result in performance degradation due to mutual co-channel interference of co-existing cellular and D2D users. Resource sharing in a D2D-based cellular network for wireless industrial applications is investigated in this paper. The presented schemes aim to maximise the overall system throughput while maintaining the Quality of Service / Experience (QoS/ QoE) requirements. Interference is managed by jointly considering admission and power control based on the relative distance between devices. A priced-Deferred Acceptance (p-DA) scheme with an incentive-based stability is developed to match D2D users to cellular resources. Numerical simulations show that the p-DA scheme is able to achieve better performance than the conventional DA algorithm and is close in performance to the presented centralised optimisation approach.
\end{abstract}

Keywords- URLLC, Distributed Algorithm, Game Theory, Radio Spectrum Management, $5 G$ and beyond.

\section{INTRODUCTION}

Ultra-Reliable Low Latency Communication (URLLC) is one of the important use cases for $5 \mathrm{G}$ and beyond technologies in addition to massive machine-type communication (mMTC) and enhanced mobile broadband (eMBB) [1]. URLLC systems support real-time communications and mission-critical applications such as factory automation and control for Factories of the Future (FoF), autonomous vehicles and remote surgery. These applications have strict Quality of Service (QoS) and Quality of Experience (QoE) requirements in terms of latency and reliability. Reliability in this context refers to guaranteed message delivery within the maximum allowable latency value and is typically expressed as a target maximum block error rate (BLER) or packet error ratio (PER) depending on the layer of the communication protocol stack. 5G and beyond wireless technologies are expected to provide performance improvements to enable these applications and services [2].

The manufacturing industry is regarded as one of the most demanding URLLC use cases with latencies typically in the order of milliseconds and a reliability figure of at least $99.999 \%$ (translated as a PER not higher than $10^{-5}$ ) [2]. The inconsistency between the growth in the number of smart devices and constraints on bandwidth for industrial wireless networks is becoming increasingly apparent in smart factories [3]. Achieving ultra-high reliability and ultra-low latency pose challenges in terms of bandwidth requirements. The scarcity of radio resources and the limitations on the available system bandwidth make spectrum sharing a necessity [4].

Device-to-Device (D2D) communication systems have been introduced a promising solution for URLLC uses-cases, especially for proximity communication (e.g. machine-type communication within a factory [5]). Factory automation typically involves closed-loop control systems that comprise a number of sensors and actuators (e.g. a robot) and a central controller (e.g. programmable logic controller, PLC). Typically, a sensor sends its measured data to a PLC, which then instructs an actuator to implement a certain action [6]. These devices have the potential of benefiting from direct device-to-device communication because they usually operate in proximity to each other. With a D2D system, proximal devices can bypass the network infrastructure and communicate directly. Integrating D2D into future industrial wireless networks and next-generation manufacturing (also referred to as Industry 4.0, smart industry or FoF), can support the creation of massive machine-type connections [7]. In addition to supporting URLLC communication requirements, a D2D communication system can improve spectrum and energy efficiencies as well as the overall network capacity [8].

D2D communication links deployed in the licensed spectrum bands have gained a lot of attention because of their reliability in terms of QoS guarantees that can be attained in comparison to the case of unlicensed bands which are unregulated and uncoordinated. Spectrum access by D2D links in a licensed band can either be implemented using shared channels with cellular users or alternatively using dedicated channels. Spectrum sharing is receiving increased interest due to the high cost associated with the license fees and the scarcity of dedicated radio resources [9]. Furthermore, spectrum efficiency in $5 \mathrm{G}$ systems will depend on developing sharing techniques to offload the already congested network and expand capacity. The main challenge to spectrum sharing schemes is how to deal with mutual (co-channel) interference. Therefore, new spectrum sharing schemes are essential to satisfy target QoS / QoE requirements of coexisting users. To maximise user density while mitigating interference in a wireless industrial network, appropriate radio resource management solutions need to be developed for such ultra-dense wireless industrial networks.

Centralised optimisation methods have gained popularity over time and have been widely explored by researchers to address the above-mentioned D2D challenges in many wireless networks. Radio resource management for D2D-based vehicular communications was considered in [10] and [11]. In [13]10, the authors proposed a heuristic centralised scheme for maximising the sum rate of cellular users by transforming the reliability and latency requirements of vehicle-to-vehicle 
communication (V2V) into computable optimisation constraints. The authors in [11] designed a minimum vehicleto-infrastructure (V2I) capacity maximisation framework to improve the overall throughput. A joint mode selection, power and spectrum allocation were proposed in [12] with the aim of maximising the total sum rates for varying network load.

The large-scale nature of the target environments and the introduction of increased numbers of Internet of Things (IoT) devices such as in smart factories pose a limitation on the adoption of a centralised approach because a large amount of information exchange is often required leading to increased complexity and signalling overheads [13]. Since IoT environments are characterised with some level of intelligence, a distributed resource management mechanism that supports self-organisation and more specifically self-optimisation is required.

Game theory approaches such as "matching game", also known as the stable matching problem or the stable marriage problem (SMP) provide a framework that allows devices and entities to make some form of interdependent choices. SMP has the potential of achieving a distributed resource allocation scheme resulting in an efficient self-organising network (SON). In SMP, each player (i.e. in our context a user equipment (UE) in a wireless network) can define their own utility function based on the local information they have from other players [14]. The deferred acceptance (DA) algorithm is often used to solve SMP [15]. The DA based algorithm can be implemented as a distributed solution with local information without requiring a centralised controller or coordinator [14]. Matching game algorithms have been applied to address resource allocation problems in D2D-based networks [16-21]. In [16], the authors used the DA algorithm to associate D2D pairs to cellular resources and introduced the concept of 'cheating' to allow further improvements for D2D pairs. The authors in [17] developed a proximity-based matching game to maximise system rate but did not consider the actual channel condition. A constrained-DA algorithm was proposed in [18] to assign preallocated macrocell users subchannels to multiple D2D users and remote radio head (RRH) users. The authors in [19] proposed a decentralised spectrum allocation scheme to maximise the D2D sum-rate but only focused on protecting the cellular users from interference posed by the D2D transmitter. The interference from a base station to a $\mathrm{D} 2 \mathrm{D}$ receiver was not considered. In [17-19], no power control was implemented.

In this paper, we investigate and compare centralised and distributed resource allocation schemes for a D2D-enabled cellular network with the aim of maximising the overall system throughput in an industrial environment targeting factories of the future (FoF). We present a model consisting of D2D users (DUEs), which are industrial devices enabled for direct D2D communication, and cellular users (CUEs). The D2D links are to share channel resources with the cellular users given that the target QoS requirements are satisfied. The CUE and DUE links are characterised by minimum Signal-to-Interference-plusNoise-Ratio (SINR) requirements to guarantee a minimum data rate. Additionally, the DUEs have minimum reliability requirements which are ensured by maintaining the outage probability below a certain threshold. The resource allocation problem is first formulated using a centralised optimisation approach namely a Mixed Integer Non-linear Programming (MINLP) problem which is NP-hard and cannot be solved directly. A distributed approach is then introduced based on the SMP framework. The problem is formulated as a one-to-one two-sided matching game and the DA algorithm is used to find a stable matching between admitted DUEs and CUEs. We then develop a priced DA ( $p$-DA) algorithm with incentive-based stability to improve resource-sharing and D2D access rate. The centralised approach assumes the knowledge of channel state information (CSI) of all links at the base station. Therefore, the centralised optimisation algorithm is considered to be the upper-bound in terms of signalling overheads. The centralised approach can, however, give an optimal performance compared to the distributed approach [12] and is therefore used as the reference scheme for comparison. The main contributions of the paper can be summarised as follows:

- We jointly consider admission and power control to determine the feasible sets of CUE-DUE pair that meet the minimum QoS requirements. To guarantee the minimum SINR requirement, co-channel interference of the CUEs and DUEs is mitigated based on the distance between the signal link and interfering link so that those users resulting in large interference are avoided. To increase the feasibility of obtaining potential sets of CUE-DUE pair, the set of power pairs that satisfy the distance metric for potential reuse partners are first obtained. Subsequently, the set of power pairs that guarantees the reliability requirement of the DUEs are then obtained. The optimal power allocation that maximises the sum throughput of the CUE and DUE is finally identified.

- To assign DUEs to cellular resources with guaranteed minimum QoS, we present a matching game solution using the priced-DA (p-DA) algorithm with an incentive-based stability to improve the resource sharing. A centralised approach is developed and used as a baseline for comparison as well as DA and random resource allocation algorithms.

- Numerical analysis is used to compare and evaluate the performance of the different presented schemes in terms of computational complexity and signalling overheads

The rest of the paper is organised as follows: In Section II, we present the system model and formulate the problem. In Section III, we discuss the resource allocation problem and present different schemes. Examples of numerical analysis and simulation results are presented in Section IV. Finally, the main conclusions are presented in Section V.

\section{THE SYSTEM MODEL}

We consider the uplink of a D2D-enabled cellular network for an industrial factory scenario as shown in Fig. 1. The network is composed of $N$ cellular users (CUEs) and $M$ D2D users (DUEs) deployed randomly. The DUEs are sensors/actuators which are enabled for direct D2D communication and are deployed along the production lines or placed on the robots. The UEs are considered to be static or quasi-static. $C=$ $\left\{c_{1}, \ldots, c_{i}, \ldots c_{N}\right\}$ denotes the set of CUEs with minimum SINR threshold values to guarantee the data rate requirements. The DUEs are URLLC links denoted by the set $D=$ $\left\{d_{1}, \ldots, d_{j}, \ldots d_{M}\right\}$ with minimum SINR and reliability requirements. The reliability constraint is guaranteed by controlling the outage probability through setting the received SINR to a minimum target value.

The network is served by a set of orthogonal sub-channels which are pre-assigned to the CUEs but can be reused by the DUE links once the minimum QoS requirements of CUEs and DUEs are jointly satisfied. We assume one-to-one spectrum reuse between DUEs and CUEs in uplink frequency division multiplexing (FDD). Interference generated by DUE transmitters in the uplink resource-sharing mode only affects the base station.

The propagation channel gains for the different links can be expressed as follows: 


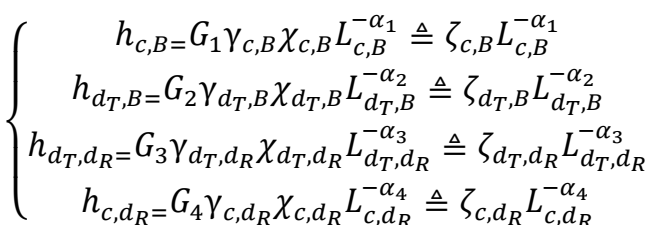

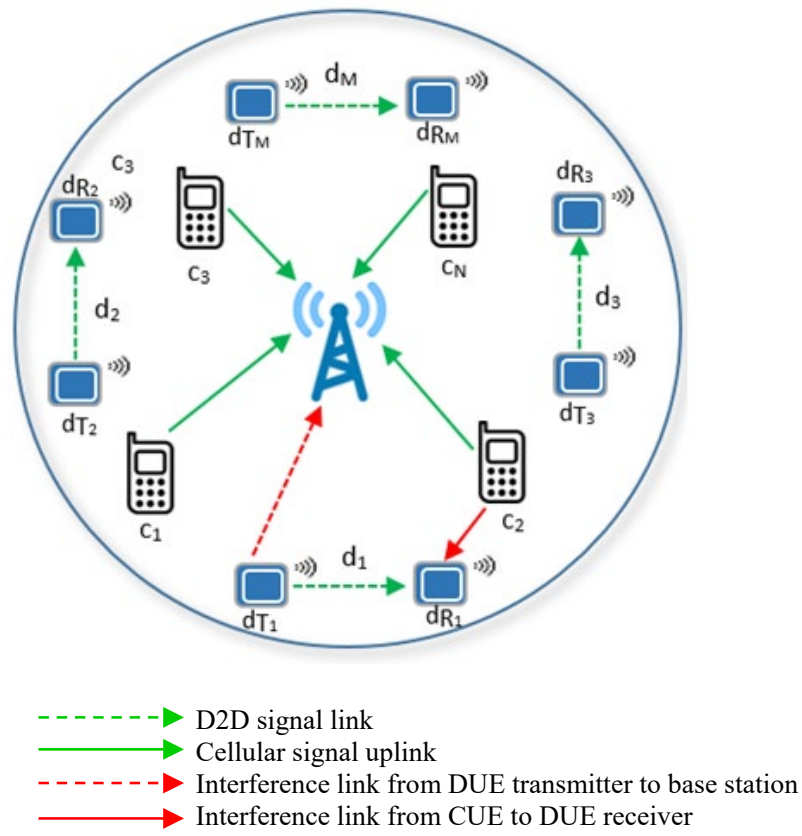

Fig.1. An Illustration of a D2D-enabled cellular network

where $h_{c, B}$, is the channel gain from CUE $c_{i}$ to the macro-base station (MBS), $B$. The channel gain from DUE link $d_{j}$ of transmitter $d_{T}$ to the receiver $d_{R}$ is $h_{d_{T}, d_{R}}$, the channel gain of the interference link from $d_{T}$ to the base station is $h_{d_{T}, B}$ and from CUE $c_{i}$ to DUE $d_{j}$ receiver is $h_{c, d_{R}} . G_{k}$ is the pathloss constant determined by system parameters, $\gamma_{q, r}$ is the smallscale fading gain due to multipath propagation and assumed to have an exponential distribution with unit mean. The largescale fading is composed of pathloss with exponent $\alpha_{k}$ and shadowing which has a slow fading gain $\chi_{q, r}$ with a log-normal distribution. $L_{q, r}$ is the distance from terminal $q$ to terminal $r$. The received SINR at the base station B from CUE $c_{i}$ and at the DUE $d_{j}$ receiver $d_{R}$ is as follows:

$$
\begin{gathered}
\Gamma_{c_{i}}=\frac{P_{c_{i}} h_{c, B}}{\sigma^{2}+\sum_{d_{j} \in D} \lambda_{j}^{i} P_{d_{j}} h_{d_{T}, B}} \\
\Gamma_{d_{j}}=\frac{P_{d_{j}} h_{d_{T}, d_{R}}}{\sigma^{2}+\sum_{c_{i} \in C} \lambda_{j}^{i} P_{c_{i}} h_{c, d_{R}}}
\end{gathered}
$$

where $P_{c_{i}}$ and $P_{d_{j}}$ are the transmit powers of CUE $c_{i}$ and DUE $d_{j}$ respectively, $\sigma^{2}$ is the variance of additive white Gaussian noise of each channel. $\lambda_{j}^{i} \in\{0,1\}$ is the resource reuse indicator, $\lambda_{j}^{i}=1$ if DUE $d_{j}$ reuses CUE $c_{i}$ subchannel and otherwise is 0 .

D2D links have the potential to enhance spectrum utilisation and increase network capacity however proper interference coordination must be ensured to harvest these benefits. For efficient D2D communication, the minimum SINR and reliability requirements of a DUE must be satisfied while guaranteeing the minimum SINR of a coexisting CUE. The reliability of DUE $d_{j}$ is expressed in terms of the maximum tolerable outage probability, $p_{R_{0}}$. The outage probability constraint, $p_{R}$, is given in (4) where $\operatorname{Pr}\{$.$\} denotes the$ probability of the input and $\Gamma_{d_{j} \text {, min }}$ is the minimum target SINR for $d_{j}$.

$$
p_{R}=\operatorname{Pr}\left(\Gamma_{d_{j}} \leq \Gamma_{d_{j}, \min }\right) \leq p_{R_{0}}
$$

For a D2D-enabled cellular network with a fixed number of subchannels $K$, the optimisation objective is to maximise the overall system throughput $T$. This is formulated as follows using Shannon capacity:

$$
\max _{\lambda_{j}^{i}, P_{c_{i}, P_{c_{j}}}} T=W_{i}\left(\sum_{c_{i} \in C}\left(\log _{2}\left(1+\Gamma_{c_{i}}\right)+\sum_{d_{j} \in D^{\mathbb{E}}} \lambda_{j}^{i} \log _{2}\left(1+\Gamma_{d_{j}}\right)\right)\right)
$$

subject to

$$
\begin{array}{ll}
\Gamma_{c_{i}} \geq \Gamma_{c_{i}, \min } & \forall c_{i} \in C \\
\Gamma_{d_{j}} \geq \Gamma_{d_{j}, \min } & \forall d_{j} \in D^{\mathbb{E}} \\
\lambda_{j}^{i} p_{R} \leq p_{R_{0}} & \forall d_{j} \in D^{\mathbb{E}} \\
\mathrm{P}_{c_{i}} \leq \mathrm{P}_{c_{i}, \max } & \forall c_{i} \in C \\
\mathrm{P}_{d_{j}} \leq \mathrm{P}_{d_{j}, \max } & \forall d_{j} \in D^{\mathbb{E}} \\
\sum_{c_{i} \in C} \lambda_{j}^{i} \leq 1 & \forall d_{j} \in D^{\mathbb{E}} \\
\sum_{d_{j} \in D^{\mathbb{E}}} \lambda_{j}^{i} \leq 1 & \forall c_{i} \in C
\end{array}
$$

where $W_{i}$ is the bandwidth, $D^{\mathbb{E}}\left(D^{\mathbb{E}} \subseteq D\right.$ ) denotes the set of admissible DUEs, $\Gamma_{c_{i} \text {, min }}$ is the minimum SINR for $c_{i} . \mathrm{P}_{c_{i}, \max }$ and $\mathrm{P}_{d_{j} \text {, max }}$ denote the maximum transmit powers of $c_{i}$ and $d_{j}$ respectively. Constraints 5(a) and 5(b) are the minimum SINR requirements for $c_{i}$ and $d_{j}$ respectively. Constraints $5(\mathrm{c})$ is the outage requirement for a valid matching between $c_{i}$ and $d_{j}$. Constraints 5(d) and 5(e) are to guarantee that the transmit of powers of $c_{i}$ and $d_{j}$ lie within the acceptable limits. Constraints 5(f) and 5(g) ensure a one-to-one assignment between CUEs and DUEs.

The optimisation problem in (5) is a Mixed Integer Non-linear Programming (MINLP) which is NP-hard. Direct solution to this problem is difficult to obtain. The problem is therefore solved by decomposing it into sub-problems as described in the following section

\section{The Resource Allocation Problem}

The network throughput optimisation problem presented above is solved by dividing it into two sub-problems. In the first step, the admission and power control are jointly solved to determine whether $c_{i}$ and $d_{j}$ are potential resource-sharing partners. The optimal power allocation that maximises the sum throughput is then obtained. Subsequently, the matching between the CUEs and DUEs is implemented to find optimal reuse partners.

\section{A. Joint Power and Admission Control}

The optimisation problem in (5) is solved by first determining whether a DUE is admissible by a CUE.

For a DUE $d_{j}$ to share resources with a CUE $c_{i}$, constraints 5(a) to 5(e) must be satisfied. Relaxing the channel assignment constraints, we have the following:

$$
\begin{aligned}
& \Gamma_{c_{i}}=\frac{P_{c_{i}} \zeta_{c, B} L_{c, B}^{-\alpha_{1}}}{\sigma^{2}+P_{d_{j}} \zeta_{d_{T}, B} L_{d_{T}, B}^{-\alpha_{2}}} \geq \Gamma_{c_{i}, \text { min }} \\
& \Gamma_{d_{j}}=\frac{P_{d_{j}} \zeta_{d_{T}, d_{R}} L_{d_{T}, d_{R}}^{-\alpha_{3}}}{\sigma^{2}+P_{d_{j}} \zeta_{c, d_{R}} L_{c, d_{R}}^{-\alpha_{4}}} \geq \Gamma_{d_{j}, \text { min }} \\
& p_{R}=\operatorname{Pr}\left(\Gamma_{d_{j}} \leq \Gamma_{d_{j}, \min }\right) \leq p_{R_{0}} \\
& \mathrm{P}_{c_{i}} \leq \mathrm{P}_{c_{i} \text {, max }}, \mathrm{P}_{d_{j}} \leq \mathrm{P}_{d_{j} \text {, max }}
\end{aligned}
$$

Equations 6(a) and 6(b) indicate that the received SINR at the base station $\mathrm{B}$ and at $d_{T}$ must be greater than minimum target threshold values. Considering the relative distances between all participating devices and base station:

$$
\left\{\begin{array}{c}
L_{d_{T}, B} \geq \Delta_{1}\left(L_{c, B}\right)^{\frac{\alpha_{1}}{\alpha_{2}}} \\
L_{c, d_{R}} \geq \Delta_{2}\left(L_{d_{T}, d_{R}}\right)^{\frac{\alpha_{3}}{\alpha_{4}}}
\end{array}\right.
$$

where 


$$
\left\{\begin{array}{c}
\Delta_{1}=\left(\frac{P_{d_{j}} \Gamma_{c_{i}, \min } \zeta_{d_{T}, B}}{P_{c_{i}} \zeta_{c, B}-\sigma^{2} \Gamma_{c_{i}, \min }\left(L_{c, B}\right)^{\alpha_{1}}}\right)^{\frac{1}{\alpha_{2}}} \\
\Delta_{2}=\left(\frac{\Gamma_{d_{j}, \min }\left[P_{c_{i}} \zeta_{c, d_{R}}+\sigma^{2}\left(L_{c, d_{R}}\right)^{\alpha_{3}}\right]}{P_{c_{i}} \zeta_{d_{T}, d_{R}}}\right)^{\frac{1}{\alpha_{4}}}
\end{array}\right.
$$

Setting $\alpha_{1}=\alpha_{2}$ and $\alpha_{3}=\alpha_{4}$, (7) becomes:

$$
\left\{\begin{array}{c}
L_{d_{T, B}} \geq \Delta_{1} L_{c, B} \\
L_{c, d_{R}} \geq \Delta_{2} L_{d_{T}, d_{R}}
\end{array}\right.
$$

From (8) we can infer that the distance of the interfering link should be greater than the distance of the intended signal link. Therefore, to mitigate interference and guarantee the minimum SINR requirements of $c_{i}$ and $d_{j}$, the relative distance between DUE $d_{j}$ transmitter $d_{T}$ and the base station B should be greater than the distance between the CUE $c_{i}$ and the base station B. Similarly, CUE $c_{i}$ should be at a larger distance from DUE $d_{j}$ compared to the distance of the DUE link.

We next determine the power allocations for which (7) is valid. The power pair extrema values that can be allocated to $c_{i}$ and $d_{j}$ while satisfying (7) are given in (9). For $\Gamma_{c_{i} \text { min }}$ to be satisfied, the minimum transmit power of $c_{i}$ and the maximum transmit power of $d_{j}$ are expressed in 9(a) and 9(b). Similarly, for the SINR threshold $\Gamma_{d_{j} \text {,min }}$ to be guaranteed, the minimum transmit power of $d_{j}$ and the maximum transmit power $c_{i}$ are expressed in $9(\mathrm{c})$ and $9(\mathrm{~d})$.

$$
\begin{aligned}
\mathrm{P}_{c_{i}, \min } & =\frac{\Gamma_{c_{i}, \min }\left(\sigma^{2}+h_{d_{T}, B} \mathrm{P}_{d_{j}, \max }\right)}{h_{c, B}} \\
\mathrm{P}_{d_{j}, \max }^{c} & =\frac{h_{c, B} \mathrm{P}_{c_{i}, \max }-\sigma^{2} \Gamma_{c_{i}, \min }}{h_{d_{T}, B} \Gamma_{c_{i}, \min }} \\
\mathrm{P}_{d_{j}, \min } & =\frac{\Gamma_{d_{j}, \min }\left(\sigma^{2}+h_{c, d_{R}} \mathrm{P}_{c_{i}, \max }\right)}{h_{d_{T} d_{R}}} \\
\mathrm{P}_{c_{i}, \max }^{d} & =\frac{h_{d_{T} d_{R}} \mathrm{P}_{d_{j}, \max }-\sigma^{2} \Gamma_{d_{j}, \text { min }}}{h_{c, d_{R}} \Gamma_{d_{j}, \min }}
\end{aligned}
$$

$P_{C}$ and $P_{D}$ denote the set of transmit extrema power values for $c_{i}$ and $d_{j}$ respectively.

$$
\begin{gathered}
P_{C}=\left\{\mathrm{P}_{c_{i} \text {,max }}, \mathrm{P}_{c_{i} \text {,max }}^{d}, \mathrm{P}_{c_{i}, \text { min }}\right\} \\
P_{D}=\left\{\mathrm{P}_{d_{j}, \text { max }}, \mathrm{P}_{d_{j}, \text { max }}^{c}, \mathrm{P}_{d_{j}, \text { min }}\right\}
\end{gathered}
$$

The possible set of power pairs for $c_{i}$ and $d_{j}$ to share the same sub-channel is the Cartesian product of $P_{C}$ and $P_{D}$ and expressed in (11) as

$$
\begin{gathered}
P_{C D}=P_{D} \times P_{c} \triangleq \\
\left\{\left(\mathrm{P}_{c_{i}, \text { max }}, \mathrm{P}_{d_{j}, \text { max }}\right),\left(\mathrm{P}_{c_{i}, \text { max }}, \mathrm{P}_{d_{j}, \text { max }}^{c}\right), \ldots,\left(\mathrm{P}_{c_{i}, \text { min }}, \mathrm{P}_{d_{j}, \text { min }}\right)\right\}
\end{gathered}
$$

The invalid power pairs are eliminated from $P_{C D}$ so that $6(\mathrm{~d})$ can be satisfied. A power pair is invalid if any of the transmit powers in the pair exceed $\mathrm{P}_{c_{i}, \max }, \mathrm{P}_{d_{j} \text {,max }}$. This is due to the dependence of the transmit powers in (9) on the channel conditions. For example, if the interference power is higher than channel gain, $\mathrm{P}_{c_{i}, \mathrm{~min}}$ tends to rise to compensate for the poor channel condition in order to meet the minimum SINR requirement [22].

Let $P_{C D}^{\tilde{C D}}$ denote the set of invalid power pairs, $P_{C D}^{\sim} \subset P_{C D}: \forall$ $\left(\mathrm{P}_{c_{i}}, \mathrm{P}_{d_{j}}\right) \in P_{C D}, \mathrm{P}_{c_{i}}>\mathrm{P}_{c_{i}, \text { max }}$ or $\mathrm{P}_{d_{j}}>\mathrm{P}_{d_{j}, \text { max }}$ or both and $P_{C D}^{-}$ denote the set of valid power pairs.

$$
P_{C D}^{-}=P_{C D}-P_{C D}^{\sim} \triangleq\left\{\left(P_{c_{i}}^{-}, P_{d_{j}}^{-}\right)_{1}, \ldots,\left(P_{c_{i}}^{-}, P_{d_{j}}^{-},\right)_{F}\right\}
$$

where $F=\left|P_{C D}^{-}\right|$.

Having obtained the set of valid power pairs, we proceed to obtain the set of power pairs for which the minimum SINR threshold values for $c_{i}$ and $d_{j}$ are guaranteed as described in Lemma 1.
Lemma 1. Denoting $f_{1}\left(\mathrm{P}_{c^{\prime}}, \mathrm{P}_{d_{j}}\right) \triangleq \Delta_{1}\left(L_{c, B}\right)^{\frac{\alpha_{1}}{\alpha_{2}}}$ and $f_{2}\left(\mathrm{P}_{c_{i}}, \mathrm{P}_{d_{j}}\right) \triangleq \Delta_{2}\left(L_{d_{T}, d_{R}}\right)^{\frac{\alpha_{3}}{\alpha_{4}}}, P_{C D}^{\prime}$ is the set of power pairs for which the minimum SINR of $c_{i}$ and $d_{j}$ are satisfied, where $G=\left|P_{C D}^{\prime}\right|$.

$$
P_{C D}^{\prime} \triangleq\left\{\left(P_{c_{i}}^{\prime}, P_{d_{j}}^{\prime}\right)_{1}, \ldots,\left(P_{c_{i}}^{\prime}, P_{d_{j}}^{\prime},\right)_{G}\right\}=\left\{\begin{array}{c}
L_{d_{T}, B} \geq f_{1}\left(\mathrm{P}_{c_{i}}, \mathrm{P}_{d_{j}}\right) \\
L_{c, d_{R}} \geq f_{2}\left(\mathrm{P}_{c_{i}}, \mathrm{P}_{d_{j}}\right) \\
\left(\mathrm{P}_{c_{i}}, \mathrm{P}_{d_{j}}\right) \in P_{C D}^{-}
\end{array}\right.
$$

The reliability constraint 6(c) is next evaluated. Reliability is expressed in terms of the outage probability. The outage probability of DUE $d_{j}$ is defined as the probability that the received SINR $\Gamma_{d_{j}}$, falls below the minimum SINR, $\Gamma_{d_{j}, \text { min }}$ and expressed as [23].

$\operatorname{Pr}\left(\Gamma_{d_{j}} \leq \Gamma_{d_{j}, \text { min }}\right)=\int_{0}^{\Gamma_{d_{j}, \text { min }}} f_{\gamma}\left(\Gamma_{d_{j}}\right) d \Gamma_{d_{j}}=F_{\gamma}\left(\Gamma_{d_{j}, \text { min }}\right)$

where $f_{\gamma}($.$) and F_{\gamma}($.$) denote the probability distribution$ function (PDF) and cumulative distribution function (CDF) respectively. The outage probability of DUE $d_{j}$ conditioned on the selected CUE $c_{i}$ is expressed as:

$$
\begin{aligned}
p_{R}= & \operatorname{Pr}\left(\Gamma_{d_{j}} \leq \Gamma_{d_{j}, \min }\right) \\
& =1-\frac{{ }_{d_{j}} g_{d_{T}, d_{R}} \exp \left(-\frac{\Gamma_{d_{j}, m_{i n} \sigma^{2}}}{\mathrm{P}_{d_{j}} g_{d_{T}, d_{R}}}\right)}{\mathrm{P}_{d_{j}} g_{d_{T}, d_{R}}+\Gamma_{d_{j}, \min \mathrm{P}_{c_{i}} g_{c_{n}, d_{R}}}} \leq p_{R_{0}}
\end{aligned}
$$

where $g_{d_{T}, d_{R}}=G_{3} \chi_{d_{T}, d_{R}} L_{d_{T}, d_{R}}^{-\alpha_{3}}$ and $g_{c, d_{R}}=G_{4} \chi_{c, d_{R}} L_{c, d_{R}}^{-\alpha_{4}}$. It is assumed that $\gamma_{c, B}$ and $\gamma_{d_{T}, d_{R}}$ are independent and identically distributed (i.i.d) exponential random variables.

Having obtained the set of power pairs, $P_{C D}^{\prime}$, for which the minimum SINR of $c_{i}$ and $d_{j}$ is satisfied in (13), the solution to the reliability constraint of $d_{j}$ is expressed using the following Lemma.

Lemma 2. Denoting $f_{3}\left(\mathrm{P}_{c_{i}}, \mathrm{P}_{d_{j}}\right) \triangleq p_{R}$, the set of power pairs, $P_{C D}^{R}$ that satisfies the minimum outage probability of $d_{j}$, for a pairing between $c_{i}$ and $d_{j}$ with minimum SINR requirement can be expressed as

$P_{C D}^{R} \triangleq\left\{\left(P_{c_{i}}^{R}, P_{d_{j}}^{R}\right)_{1}, \ldots,\left(P_{c_{i}}^{R}, P_{d_{j}}^{R}\right)_{H}\right\}=\left\{\begin{array}{c}f_{3}\left(\mathrm{P}_{c_{i}}, \mathrm{P}_{d_{j}}\right) \leq p_{R_{0}} \\ \left(\mathrm{P}_{c_{i}}, \mathrm{P}_{d_{j}}\right) \in P_{C D}^{\prime}\end{array}\right.$

where $H=\left|P_{C D}^{R}\right|$. The minimum QoS of $c_{i}$ and $d_{j}$ can be satisfied from (16) and expressed using Lemma 3.

Lemma 3. CUE $c_{i}$ and DUE $d_{j}$ are potential resource-sharing partners if $P_{C D}^{R} \neq \emptyset$.

Having addressed the admission and power control to satisfy the minimum QoS requirement for a pairing between $c_{i}$ and $d_{j}$, we then proceed to evaluate the optimal transmit powers for CUE $c_{i}$ and DUE $d_{j}$ that maximise the sum throughput. This can be expressed as follows:

$\left(P_{c_{i}}^{*}, P_{d_{j}}^{*}\right)=\underset{\mathrm{P}_{c_{i}} \mathrm{P}_{d_{j}}}{\arg \max _{j}}\left[W_{i}\left(\log _{2}\left(1+\Gamma_{c_{i}}\right)+\log _{2}\left(1+\Gamma_{d_{j}}\right)\right)\right]$

subject to $(6 a)-(6 d)$

The optimisation problem in (17) aims at finding the power pairs within the set $P_{C D}^{R}$, that maximise the sum throughput of $c_{i}$ and $d_{j}$ and expressed in the following Lemma.

Lemma 4. Denoting $f_{4}\left(\mathrm{P}_{c_{i}}, \mathrm{P}_{d_{j}}\right) \triangleq W_{i}\left(\log _{2}\left(1+\Gamma_{c_{i}}\right)+\right.$ $\left.\log _{2}\left(1+\Gamma_{d_{j}}\right)\right)$, the optimal power pair $\left(P_{c_{i}}^{*}, P_{d_{j}}^{*}\right)$ in (17) is:

$$
\left(P_{c_{i}}^{*}, P_{d_{j}}^{*}\right)=\arg \max _{\left(\mathrm{P}_{c_{i}}, \mathrm{P}_{d_{j}}\right) \in P_{C D}^{R}} f_{4}\left(\mathrm{P}_{c_{i}}, \mathrm{P}_{d_{j}}\right)
$$

From the above Lemma, it is shown that for a pair $\left(P_{c_{i}}^{*}, P_{d_{j}}^{*}\right)$, either the CUE or DUE has to transmit at peak power to 
maximise the sum throughput as will be also shown in the example simulation results below.

\section{B. Matching Between CUEs and DUES}

After identifying the resource-sharing pairs satisfying minimum QoS requirements and the optimal power allocations to maximise sum throughput, we now aim at finding the optimal reuse partner for the CUEs $\forall c_{i} \in C$ with multiple reuse candidates. Let $R_{c_{i}}^{d}$ be the set of reuse (or admissible) DUEs for CUE $c_{i}$ and $R_{d_{j}}^{c}$ be the set of CUEs that $d_{j}$ can share resources with.

We refer to CUEs (DUEs) with $R_{c_{i}}^{d} \neq \varnothing\left(R_{d_{j}}^{c} \neq \varnothing\right)$ as eligible CUEs (admissible DUEs). The set of all admissible DUEs, $\quad D^{\mathbb{E}}=\left\{R_{c_{1}}^{d} \cup R_{c_{i}}^{d} \cup \ldots \cup R_{c_{N}}^{d}\right\} \triangleq\left\{d_{1}, \ldots, d_{j}, \ldots, d_{m}\right\}$ where $m=\left|D^{\mathbb{E}}\right|$ whereas the set of all eligible CUEs, $C^{\mathbb{E}}=$ $\left\{R_{d_{1}}^{c} \cup R_{d_{2}}^{c} \cup \ldots \cup R_{d_{M}}^{c}\right\} \triangleq\left\{c_{1}, \ldots, c_{i}, \ldots, c_{n}\right\}$ where $n=\left|C^{\mathbb{E}}\right|$. The resource allocation constraint for multiple eligible CUEs and admissible DUEs is given as

$$
\begin{cases}\sum_{d_{j} \in D^{\mathbb{E}}} \lambda_{j}^{i} \leq 1 & \forall c_{i} \in C^{\mathbb{E}} \\ \sum_{c_{i} \in C^{\mathbb{E}}} \lambda_{j}^{i} \leq 1 & \forall d_{j} \in D^{\mathbb{E}}\end{cases}
$$

To identify the optimal resource-sharing partners, we present two main approaches: centralised and distributed. In the centralised approach, the base station requires the full acquisition of channel state information (CSI) of all links and the minimum QoS requirement of each user equipment (UE). For CUE-BS and DUE-BS links, the CSI is measured at the BS while for UE-UE links, the parameters can be estimated at the DUE receivers and reported to the BS via a control channel. This increases the amount of signalling overheads.

The distributed approach is device or user-centric. The UEs are capable of making resource management decisions based on local information without relying on a centralised controller. The DUEs measure their received SINR values based on local information. There is, therefore, no requirement for feedbacks to the BS. The BS only measures the received signal and interference power from the CUE $c_{i}$ and DUE transmitter $d_{T}$.

The joint power and admission algorithm to determine the set of eligible CUEs and admissible DUEs with guaranteed minimum QoS requirements is described in Algorithm I.

We now present and compare the different approaches for resource allocation namely centralised, random and game theoretic schemes.

\section{The centralised algorithm:}

The optimal resource sharing partner for CUE $c_{i}$ is DUE $d_{j}$ that achieves the highest sum rate. When DUE $d_{j} \in D^{\mathbb{E}}$ reuses the resource of $c_{i} \in C^{\mathbb{E}}$ is the sum throughput and expressed as

$$
T_{c_{i}, d_{j}}=W_{i}\left(\log _{2}\left[\frac{P_{c_{i}}^{*} h_{c, B}}{\sigma^{2}+P_{d_{j}}^{*} h_{d_{T}, B}}\right]+\log _{2}\left[\frac{P_{d_{j}}^{*} h_{d_{T}, d_{R}}}{\sigma^{2}+P_{c_{i}}^{*} h_{c, d_{R}}}\right]\right)
$$

where $\left(P_{c_{i}}^{*}, P_{d_{j}}^{*}\right)$ is optimal power as obtained in Lemma 4. Thus, the optimal partner of $c_{i}$ when there multiple D2D is

$$
d_{j}^{*}=\underset{d_{j} \in R_{c_{i}}^{d}}{\operatorname{argmax}} T_{c_{i}, d_{j}}
$$

For multiple DUEs and CUEs, the resource allocation procedure becomes complicated as different CUEs can have the same optimal partners and may have varying size of $R_{c_{i}}^{d}$. The resource allocation problem can be formulated as:

$$
\begin{gathered}
\max _{c_{i} \in C^{\mathbb{E}}, d_{j} \in D^{\mathbb{E}}} \lambda_{j}^{i} T_{c_{i}, d_{j}} \\
\sum_{d_{j} \in \boldsymbol{D}^{\mathbb{E}}} \lambda_{j}^{i} \leq 1 \\
\sum_{c_{i} \in C^{\mathbb{E}}} \lambda_{j}^{i} \leq 1
\end{gathered}
$$

The centralised solution for optimal resource allocation for multiple CUEs and DUEs is presented in Algorithm II. with | . | indicating the cardinality of the set.

\section{The Random allocation algorithm:}

Random matching uses the centralised approach presented above. However, no optimisation is required for the matching process which results in a reduced complexity.

Algorithm I. Joint Admission and Power Control (JAPC) to determine the set of eligible CUEs and admission DUEs with minimum QoS requirements.

1: Input: $\mathrm{C}, \mathrm{D}, \Gamma_{c_{i}, \min }, \Gamma_{d_{j}, \min }, \mathrm{P}_{c_{i}, \max }$ and $\mathrm{P}_{d_{j}, \max }$

2: Set up the set of admissible DUEs for $c_{i}, R_{c_{i}}^{d}, R_{c_{i}}^{d}=\emptyset$, $\forall c_{i} \in C$

3: Set up the set of eligible CUEs for $d_{j}, R_{d_{j}}^{c}, R_{d_{j}}^{c}=\emptyset$, $\forall d_{j} \in D$

4: for $c_{i} \in C \quad 1 \leq i \leq N$ do

5: $\quad$ for $d_{j} \in D \quad 1 \leq j \leq M$ do

6: $\quad$ compute valid set of power pair $P_{C D}^{-}$as in (12)

7: $\quad$ compute set of power pair $P_{C D}^{\prime}$, for which $\Gamma_{c_{i} \text {, min }}$ and $\Gamma_{d_{j} \text {,min }}$ is satisfied as in (13) $\leftarrow$ Lemma 1

8: $\quad$ if $P_{C D}^{\prime} \neq \varnothing$ then

9: $\quad$ compute set of power pair $P_{C D}^{R}$ for which $p_{R}$ is satisfied as in (16) $\leftarrow$ Lemma 2

10: $\quad$ if $P_{C D}^{R} \neq \varnothing$ then $\quad \leftarrow$ Lemma 3

11: $\quad d_{j}$ is a reuse candidate for $c_{i}$

12: $\quad R_{c_{i}}^{d}=R_{c_{i}}^{d}+d_{j}$

13: $\quad R_{d_{j}}^{c}=R_{d_{j}}^{c}+c_{i}$

14:

15:

16:

17:

21: end for

22: $C^{\mathbb{E}}=\left\{R_{d_{1}}^{c} \cup R_{d_{2}}^{c} \cup \ldots \cup R_{d_{M}}^{c}\right\}$

22: $D^{\mathbb{E}}=\left\{R_{c_{1}}^{d} \cup R_{c_{i}}^{d} \cup \ldots \cup R_{c_{N}}^{d}\right\}$

22: Output $C^{\mathbb{E}}, D^{\mathbb{E}}, R_{c_{i}}^{d} \forall c_{i} \in C^{\mathbb{E}}, R_{d_{j}}^{c} \forall d_{j} \in D^{\mathbb{E}}$ and $\left(P_{c_{i}}^{*}, P_{d_{j}}^{*}\right)$ for potential $\left(c_{i}, d_{j}\right)$ pairing

\section{The Stable Marriage Problem (SMP):}

The resource allocation problem is modelled using the framework of Stable Matching Problem (SMP), a one-to-one two-sided matching game theoretic approach. The players in the game are a set of eligible CUEs $C^{\mathbb{E}}$ and a set of admissible DUEs $D^{\mathbb{E}}$ with preference profiles that allow them to build their

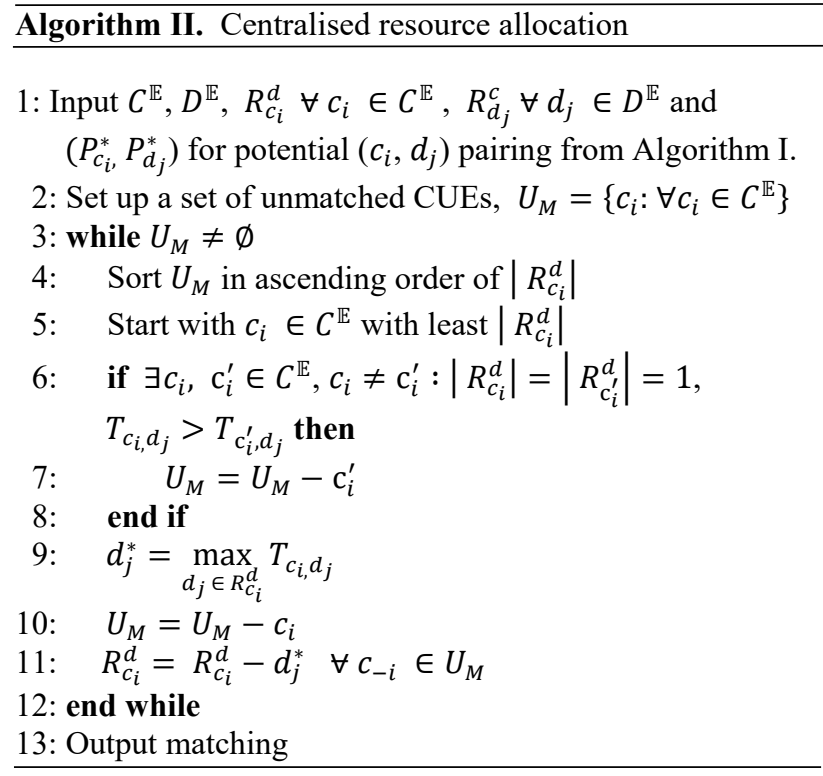


preference list. The output of the game is the matching of a CUE channel to a DUE. The definition of matching is given as follows [24]:

Definition 1: A matching $\mu$ between eligible DUEs and eligible CUEs is a function that maps the set of $\boldsymbol{D}^{\mathbb{E}}$ DUEs to set of $\boldsymbol{C}^{\mathbb{E}}$ CUEs such that:

1. $\mu\left(d_{j}\right) \in C^{\mathbb{E}} \cup\{\varnothing\}$ and $\left|\mu\left(d_{j}\right)\right| \in\{0,1\}$

2. $\mu\left(c_{i}\right) \in D^{\mathbb{E}} \cup\{\varnothing\}$ and $\left|\mu\left(c_{i}\right)\right| \in\{0,1\}$

where $\mu\left(d_{j}\right)=\left\{c_{i}\right\} \Leftrightarrow \mu\left(c_{i}\right)=\left\{d_{j}\right\}, \quad \forall d_{j} \in D^{\mathbb{E}}, \forall c_{i} \in C^{\mathbb{E}}$ and $|\mu()$.$| indicates the cardinality of the outcome. Definition$ 1 states that $\mu$ is a one-to-one matching which implies one eligible DUE can reuse only one eligible CUE subchannel and an eligible CUE can share its resource with only one eligible DUE to satisfy (19). $\mu\left(c_{i}\right), \mu\left(d_{j}\right)=\emptyset$ implies there is no resource-sharing between an eligible CUE and eligible DUE. For any DUE $d_{j} \in D^{\mathbb{E}}$ and $c_{i} \in C^{\mathbb{E}}$ such that $c_{i} \in \mu\left(d_{j}\right), c_{i}$ and $d_{j}$ prefers to be matched to each other than being unmatched.

\subsection{Utility function and preference profile}

The utility $c_{i} \in C^{\mathbb{E}}$ obtains from sharing its subchannel with DUEs $d_{j} \in R_{c_{i}}^{d}$ from is the CUE's throughput and defined as

$$
U_{c_{i}}^{d_{j}}=W_{i} \log _{2}\left(1+\frac{P_{c_{i}}^{*} h_{c, B}}{\sigma^{2}+P_{d_{j}}^{*} h_{d_{T, B}}}\right)
$$

while the utility of any eligible DUE $d_{j} \in D^{\mathbb{E}}$ obtains from reusing subchannel of CUE $c_{i} \in R_{d_{j}}^{c}$ is the DUE's throughput when matched to the CUE and defined as

$$
U_{d_{j}}^{c_{i}}=W_{i} \log _{2}\left(1+\frac{P_{d_{j}}^{*} h_{d_{T}, d_{R}}}{\sigma^{2}+P_{c_{i}}^{*} h_{c, d_{R}}}\right)
$$

Definition 2: A preference relation $>$ is a complete, reflexive and transitive binary relation between the set of CUE $c_{i} \in C^{\mathbb{E}}$ and DUE $d_{j} \in D^{\mathbb{E}} . \forall c_{i} \in C^{\mathbb{E}}$ define a strict preference relation $>_{c}$ over a set of DUEs $R_{c_{i}}^{d} \subseteq D^{\mathbb{E}}$ such that any two DUEs $d_{1}$, $d_{2} \in R_{c_{i}}^{d}, d_{1} \neq d_{2}$,

$$
d_{1}>_{c_{i}} d_{2} \Leftrightarrow U_{c_{i}}^{d_{1}}>U_{c_{i}}^{d_{2}}
$$

This implies that $c_{i}$ prefers $d_{1}$ to $d_{2} . \forall d_{j} \in D^{\mathbb{E}}$, we define a strict preference relation $>_{d}$ over a set of CUEs $R_{d_{j}}^{c} \subseteq$ such that any two CUEs $c_{1}, c_{2} \in R_{d_{j}}^{c} c_{1} \neq c_{2}$,

$$
c_{1}>_{d_{j}} c_{2} \Leftrightarrow U_{d_{j}}^{c_{1}}>U_{d_{j}}^{c_{2}}
$$

This implies that $d_{j}$ prefers $c_{1}$ and $c_{2}$.

With the preference relation, $\forall c_{i} \in C^{\mathbb{E}}$ and $\forall d_{j} \in D^{\mathbb{E}}$ can build their preference list $P L_{c_{i}}$ and $P L_{d_{j}}$ by ranking $R_{c_{i}}^{d}$ and $R_{d_{j}}^{c}$ respectively, giving priority to ones that provides higher utility. The solution to the game is a match $\mu$ defined on the set $C^{\mathbb{E}}$ and $D^{\mathbb{E}}$ that assigns a DUE to a CUE.

Definition 3: A matching $\mu$ is stable if there does not exist any pair of CUEs $c_{i}, c_{i}^{\prime} \in C^{\mathbb{E}}$ assigned respectively to DUEs $d_{j}^{\prime}, d_{j} \in$ $D^{\mathbb{E}}$, although $c_{i}$ prefers $d_{j}$ to $d_{j}^{\prime}$ i.e., $d_{j}>_{c_{i}} d_{j}^{\prime}$ and $d_{j}$ prefers $c_{i}$ to $c_{i}^{\prime}$ i.e., $c_{i}>_{d_{j}} c_{i}^{\prime}$. $\left(c_{i}, d_{j}\right)$ are said to be blocking pairs.

\subsection{Deferred Acceptance (DA)}

The deferred acceptance (DA) algorithm is often used to solve the SMP, in which each of $x$ men and $y$ women rank the members of the opposite side in order of preference. Conventionally, DA is used to solve SMP for which $x=y$ (i.e. equal sets of men and women). In DA, each $x$ proposes to $y$ on an $x$ preference list, starting with the most preferred $y$ and ceases when a proposal is considered, but continues if a proposal is rejected. When $y$ receives a proposal, it is rejected if there is a more preferable proposal, and otherwise agreed to be held for consideration. The algorithm terminates when no $x$ needs to propose [15], [16].
We assume the CUEs to be $x$ and DUEs to be $y$. In contrast with the classical SMP, where there are two equally sized sets of elements with ordering of preferences for each member of the opposite side, the size of sets in our problem under consideration depends on $n=\left|C^{\mathbb{E}}\right|$ and $m=\left|D^{\mathbb{E}}\right|$. Moreover, $\forall c_{i} \in C^{\mathbb{E}}\left(\forall d_{j} \in D^{\mathbb{E}}\right)$ we rank only the members of $R_{c_{i}}^{d}\left(R_{d_{j}}^{c}\right)$ and not set $D(\operatorname{set} C)$ to construct its preference list.

Definition 4: For $n \neq m, \exists c_{i} \in C^{\mathbb{E}}$ for which $\left|P L_{c_{i}}\right|=1=$ $\left\{d_{j}\right\}$ and $c_{i} \neq \max P L_{d_{j}}$, then $\mu\left(c_{i}\right)=\emptyset$. This states that if there exist a CUE $c_{i} \in H_{c}$ with only one potential DUE partner $d_{j}$ in its preference list, and $c_{i}$ is not the highest ranked (most preferred) by $d_{j}$ then $c_{i}$ will certainly be unmatched at the output $\mu$. Therefore, the output of the matching may not be optimal from a resource sharing point of view using the DA approach as some eligible CUE(s) might not be matched. To overcome this challenge and maximise number of eligible CUEs sharing their channels, we introduce a 'priced' Deferred Acceptance (p-DA).

\subsection{Priced Deferred Acceptance ( $p-D A)$}

We assume that each connected UE is charged with fees corresponding to the achieved data rate. Let $\pi_{c_{i}}$ and $\pi_{d_{j}}$ represent the price charged per connection for the CUEs and DUEs respectively.

$$
\left\{\begin{array}{l}
\pi_{c_{i}}=\psi W_{i} \log _{2}\left(1+\Gamma_{c_{i}}\right) \\
\pi_{d_{j}}=\psi W_{i} \log _{2}\left(1+\Gamma_{d_{j}}\right)
\end{array}\right.
$$

where $\psi$ is the price per unit rate and assumed to be uniform for all the UEs. Therefore, the total revenue generated by the $\mathrm{BS}$ is given by (28).

$$
U_{B}(\pi)=\sum_{c_{i} \in C} \pi_{c_{i}}+\sum_{d_{j} \in D^{\mathbb{E}}} \pi_{d_{j}}
$$

with $1 \leq i \leq N$ and $1 \leq j \leq D_{m}$, where $D_{m}$ is the number of admitted DUEs.

To increase the number of CUE-DUE pairing and DUE access rate (or reuse gain), $d_{j} \in D^{\mathbb{E}}$ considers the size of the preference list $P L_{c_{i}}$, of $\forall c_{i} \in C^{\mathbb{E}}$ that proposes at each iteration round and gives priority to the most preferred CUE with the least size of $P L_{c_{i}}$. This is because the larger the size of $P L_{c_{i}}$, then $c_{i} \in C^{\mathbb{E}}$ will have more DUEs to propose to after being rejected in a previous round of proposals and vice versa. In particular, at iteration $k, \forall d_{j} \in D^{\mathbb{E}}$ will consider the proposal of the most preferred CUE with least size of preference list at iteration $k+1,\left|P L_{c_{i}}{ }^{(k+1)}\right|$, defined in (29)

$$
\left|P L_{c_{i}}{ }^{(k+1)}\right|=\left|P L_{c_{i}}{ }^{(k-1)}\right|-d_{j}^{(k)}
$$

Definition 5: For $c_{i}, \mathrm{c}_{i}^{\prime}, \mathrm{c}_{i}^{\prime \prime} \in C^{\mathbb{E}}, c_{i} \neq \mathrm{c}_{i}^{\prime} \neq \mathrm{c}_{i}^{\prime \prime}$, that proposes to $d_{j}$ such that $U_{d_{j}}^{\mathrm{c}_{i}^{\prime}}>U_{d_{j}}^{c_{i}}>U_{d_{j}}^{\mathrm{c}_{i}^{\prime \prime}},\left|P L_{\mathrm{c}_{i}^{\prime}}\right|>\left|P L_{c_{i}}\right|,\left|P L_{\mathrm{c}_{i}^{\prime \prime}}\right|$ and $\left|P L_{c_{i}}\right|=\left|P L_{\mathrm{c}_{i}^{\prime \prime}}\right|, \mathrm{c}_{i}^{\prime}$ is the most preferred CUE, while $c_{i}$ is the most preferred CUE with the least size of preference list, then $d_{j}$ will consider the proposal of $c_{i}$ and reject the rest. However, $\left(\mathrm{c}_{i}^{\prime}, d_{j}\right)$ will form a blocking pair at the matching output $\mu$, if $\mathrm{c}_{i}^{\prime}$ is the highest ranked CUE that proposes to $d_{j}$ when the algorithm terminates.

To ensure stability, we present a monetised incentive-based mechanism to balance the utility loss of $d_{j}$. Since utility is in terms of the achieved rate, $d_{j}$ will demand from the $\mathrm{BS}$, a reduction in its price which is equivalent to its rate loss from being paired with $c_{i}$ rather than $c_{i}^{\prime}$ else $d_{j}$ will deviate from the matching.

$T_{d_{j},}\left[c_{i}\right]$ and $T_{d_{j}},\left[\mathrm{c}_{i}^{\prime}\right]$ denote of the achieved rate from $\left(c_{i} d_{j}\right)$ and $\left(c_{i}^{\prime} d_{j}\right)$ pairing respectively. The rate loss of $d_{j}$ is given in (30). 


$$
\tau=T_{d_{j},}\left[\mathrm{c}_{i}^{\prime}\right]-T_{d_{j},}\left[c_{i}\right]
$$

where

$$
\tau=\left\{\begin{array}{cl}
0, & c_{i}=c_{i}^{\prime} \\
T_{d_{j},}\left[c_{i}^{\prime}\right] \leq \tau<T_{d_{j},}\left[c_{i}\right], & \text { otherwise }
\end{array}\right.
$$

The price of the rate loss is given as follows:

$$
\pi_{\mathcal{L}}=\psi \tau
$$

The new rate price for $d_{j}$ for $\left(c_{i} d_{j}\right)$ pairing for that balances its rate loss and new revenue for the BS is expressed in (32) and (33) respectively.

$$
\begin{aligned}
& \pi_{d_{j}}^{*}=\pi_{d_{j}}-\pi_{\mathcal{L}} \\
& U_{B}^{*}(\pi)=\sum_{c_{i} \in C} \pi_{c_{i}}+\sum_{d_{j} \in D^{\mathbb{E}}} \pi_{d_{j}}^{*}
\end{aligned}
$$

We analyse the characteristics of the output of the p-DA matching with respect to classical DA matching in the CUEDUE pairings. In what follows, we denote $c_{i}$ as the most preferred CUE and $c_{i}^{\prime}$ as the most preferred CUE with least preference list at iteration $k$. Let $D_{M}$ be set of admitted DUE, where $D_{m}=\left|D_{M}\right| . D_{M}$ is characterised by subsets of DUEs denoted by $D_{D A}, D_{-}$and $D_{+}$. Let $c_{i}^{*}$ be the matched partner of $d_{j}$ under p-DA and $c_{i}^{-*}$ be the matched partner of $d_{j}$ under DA. Without loss of generality, we assume that $\mathrm{c}_{i}^{*}$ is matched (or makes it final proposal) at iteration $k$.

1. $D_{D A}$ is the set of DUEs under p-DA with CUE partners identical with the matching in DA: This implies $d_{j} \in \mu\left(\mathrm{c}_{i}^{*}\right)=$ $d_{j} \in \mu\left(\mathrm{c}_{i}^{-*}\right)$, hence $U_{\mathrm{c}_{i}^{*}}^{d_{j}}=U_{\mathrm{c}_{i}^{-*}}^{d_{j}}$. This happens if DUE $d_{j}$ accepts $\mathrm{c}_{i}^{*}$ because $\mathrm{c}_{i}^{*}=c_{i}=\mathrm{c}_{i}^{\prime}$ under DA and $\mathrm{c}_{i}^{-*}=\mathrm{c}_{i}^{\prime}$, thus $\mathrm{c}_{i}^{*}=\mathrm{c}_{i}^{-*}$.

2. $D_{-}$is set of DUEs under p-DA with CUE partners with utilities lower than the matched partners in DA: This implies $d_{j} \in \mu\left(\mathrm{c}_{i}^{*}\right)$ under p-DA will generate lower utility for $d_{j}$ than $d_{j} \in \mu\left(\mathrm{c}_{i}^{-*}\right)$ under DA, hence, $U_{\mathrm{c}_{i}^{*}}^{d_{j}}<U_{\mathrm{c}_{i}^{-*}}^{d_{j}}$. If CUE $\mathrm{c}_{i}^{-*}=\mathrm{c}_{i}^{\prime}$, then DUE $d_{j}$ will consider the proposal of $\mathrm{c}_{i}^{-*}$ under DA, and reject $\mathrm{c}_{i}^{-*}$ under p-DA if $\mathrm{c}_{i}^{-*} \neq c_{i}$ and be matched to $\mathrm{c}_{i}^{*}=c_{i}$ while the BS incentivise $d_{j}$ with rate loss price, $\pi_{\mathcal{L}}$ to remain matched to $\mathrm{c}_{i}^{*}$.

3. $D_{+}$is the set of DUEs with CUE partners with utilities higher than matched partners DA $\left(d_{j} \in \mu(\varnothing)\right.$ in DA inclusive): This implies $d_{j} \in \mu\left(\mathrm{c}_{i}^{*}\right)$ under p-DA generate higher utility for $d_{j}$ than $d_{j} \in \mu\left(\mathrm{c}_{i}^{-*}\right)$ under DA, thus $U_{\mathrm{c}_{i}^{*}}^{d_{j}}>U_{\mathrm{c}_{i}^{-*}}^{d_{j}}$. This happens if $\mathrm{c}_{i}^{-*}$ has been held for consideration by $d_{j}$ because $\mathrm{c}_{i}^{-*}=c_{i}$ while $\mathrm{c}_{i}^{*}$ is rejected by a DUE $d_{j}^{\prime}$ under p-DA but $\mathrm{c}_{i}^{-*}=\mathrm{c}_{i}^{\prime}$ under DA at some previous iteration $k-l$. At iteration $k, d_{j}$ will be reject $\mathrm{c}_{i}^{-*}$ and be matched to $\mathrm{c}_{i}^{*}=c_{i}=\mathrm{c}_{i}^{\prime}$.

Based on the above analysis, the rate loss price, $\pi_{l}$ paid by the $\mathrm{BS}$ as incentive to $d_{j} \in D_{-}$is offset rate price gain due to rate increase by $d_{j} \in D_{+}$.

The p-DA algorithms are summarised in Algorithms III.

Proposition 1: The matching $\mu$ returned by the p-DA algorithm is stable

Proof: The p-DA algorithm will converge to stable matching after finite number of iterations since $m$ and $n$ is finite. The stable matching for SMP under DA always exists [15]. Proposition 1 is proved by contraction. Assume that the p-DA algorithm produces a matching $\mu$ with a blocking pair $\left(c_{i}, d_{j}\right)$ then $\exists c_{i}^{\prime} \in \mu\left(d_{j}\right): c_{i}>_{d_{j}} c_{i}^{\prime}$ and $d_{j}>_{c_{i}} \mu\left(c_{i}\right)$. Thus, $c_{i}$ must have proposed to $d_{j}$. Since $c_{i}$ has proposed and not matched to $d_{j}$, it means that (i) $c_{i}$ has been rejected because it not the most preferred CUE with the least preference list. Since $c_{i}$ was rejected, $c_{i}^{\prime}$ will also be rejected as $c_{i}$ is ranked higher than $c_{i}^{\prime}$. Hence $c_{i}^{\prime} \notin \mu\left(d_{j}\right)$, which contradicts the assumption or (ii) $c_{i}$ is the most preferred CUE but $d_{j}$ is matched to $c_{i}^{\prime}$ because the rate price of $d_{j}$ has been reduced by the rate loss ratio from being matched to $c_{i}$. If $d_{j}$ rejects $c_{i}^{\prime}$, then it is matched to $c_{i}$ and stable by the DA algorithm and so a contradiction to the assumption.

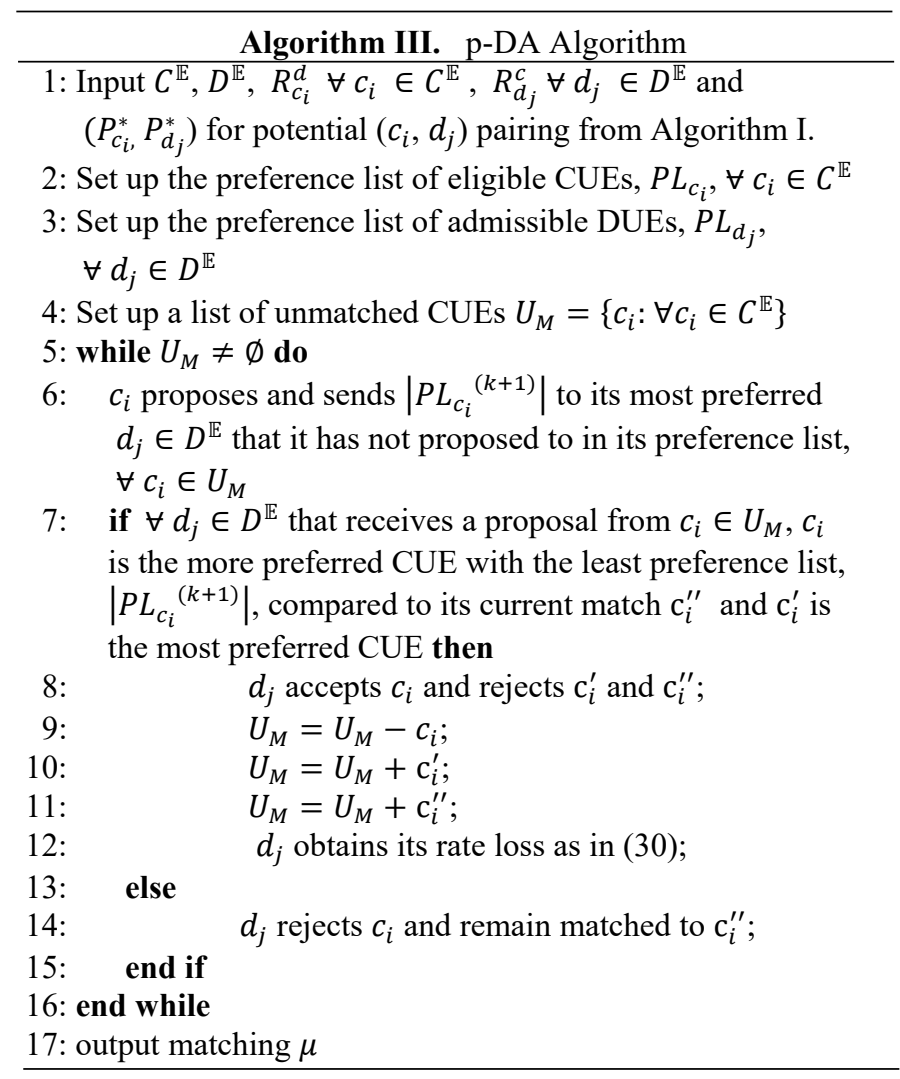

In the following section, we investigate and compare the performance of the different algorithms presented above.

\section{PERFORMANCE ANALYSIS}

\section{A. Signalling overhead and Complexity}

The information required to evaluate parameters $C^{\mathbb{E}}, D^{\mathbb{E}}$, $R_{c_{i}}^{d}\left(\forall c_{i} \in C^{\mathbb{E}}\right), R_{d_{j}}^{c}\left(\forall d_{j} \in D^{\mathbb{E}}\right)$ and implement matching include the maximum transmit power levels for $c_{i}$ and $d_{j}, \mathrm{P}_{c_{i}, \text { max }}$ and $\mathrm{P}_{d_{j}, \text { max }}$ respectively, the minimum SINR requirements and the channel gains between the links. Centralised optimisation and random approaches have large overheads as they require the CSI of all the links available at the BS, which increases as the number of UEs grows. This information includes the CSI of $N$ CUE-BS links, $M$ D2D links on $N$ channels, and interference CSI of $M d_{T}$-BS links on $N$ channels and $M$ CUE- $d_{R}$ links on $N$ channels making a total $3 N M+N$ links. For the DA and p-DA, each DUE needs to acquire the channel gains of its communication and interference link on $N$ channels. Thus, the information needed by each D2D link is $2 N$. The DUE obtains $R_{d_{j}}^{c}$ and constructs the preference list $P L_{d_{j}}$ based on the local CSI at its receiver. The received signal and interference power from $N$ CUEs and $M$ DUE transmitters respectively are measured at the BS and sent to the CUEs to evaluate $R_{c_{i}}^{d}$ and build $P L_{c_{i}}$. Few bits are exchanged to send proposals and receive acknowledgements with an accept or reject. No additional signalling is required for $\mathrm{p}-\mathrm{DA}$ as the $\left|P L_{c_{i}}{ }^{(k+1)}\right|$ is sent with the proposals at each iteration. We also note that each CUE or DUE has no knowledge of the received SINRs and preference lists of other CUEs and DUEs.

We compare the algorithms in terms of computational complexity. The complexity of Algorithm I for the JAPC algorithm has an upper bound set by $\mathrm{O}(N M)$. For the centralised optimisation matching algorithm, with $n$ eligible 
CUEs, the while-loop will be executed $n$ times. Within the while-loop, the number of eligible CUEs decreases as a CUE is assigned at each round of iteration in the sequence $n, n-$ $1, \ldots, 1$. The sequence is dominated $n$ and has a sorting complexity of $\mathrm{O}\left(n^{2}\right)$. Thus, Algorithm II has a worst-case complexity of $\mathrm{O}\left(n^{3}\right)$. The random matching algorithm has a worst-case main runtime complexity of $\mathrm{O}(n)$. For DA and pDA algorithm, the complexity to construct the preference profile for standard sorting algorithm for CUEs and DUEs is $\mathrm{O}\left(\mathrm{nm}^{2}\right)$ and $\mathrm{O}\left(\mathrm{mn}^{2}\right)$ for worst case scenario respectively. The complexity for the matching subroutine is $\mathrm{O}(\mathrm{nm})$. The overall computational complexity for DA and p-DA matching algorithm is $\mathrm{O}\left(n m^{2}+m n^{2}+n m\right)$ in the worst case. For scenario $N=M=50$, with $n=12$ and $m=10$, the DA algorithm was approximately $10 \%$ faster in run-time on our platform compared to the centralised matching approach.

In the following section, we investigate and compare the performance of the different algorithms presented above.

\section{B. Example Simulation Results}

We present examples of simulation results carried out to validate and compare the presented algorithms. We simulate an industrial environment with a factory floor dimensions of $300 \mathrm{~m} \times 300 \mathrm{~m}$. We consider the uplink of a single-cell LTE-A wireless network. The base station has a $400 \mathrm{~m}$ cell radius, positioned at the center of the factory layout. $N$ CUEs are randomly distributed within the cell coverage and the $M$ DUEs or factory devices (i.e. sensors and/or actuators enabled for D2D communications links) are also randomly distributed within the factory floor. For communication between the DUEs, the channel is modeled using the A1-Indoor scenario of the Winner II project [25]. The main simulation parameters are summarised in Table I and the summary of the channel models for the CUE and DUE links are given in Table II.

We assume that each CUE has been pre-allocated at most one sub-channel. The metrics for evaluating the performance of the algorithms are the achieved throughput and number of admitted DUEs, $D_{m}$, which corresponds to the number of shared channels. The admitted DUEs can reuse cellular radio resources with a guaranteed reliable communication. The centralised approach is used as a reference or baseline algorithm for comparison with the other approaches.

First, we evaluate the impact of varying the outage probability of the DUEs, $p_{R_{0}}$, on the number of admissible DUEs, $n$ using full CSI. As seen in Fig. 2, $n$ gets larger as higher $p_{R_{0}}$ is allowed. This can be attributed to the fact higher outage probabilities of the DUEs make them more tolerant of interference from the CUEs, thus enabling more CUEs to find potential partners for resource sharing.

In Fig. 3, we compare the number of admitted DUEs, $D_{m}$, for the four algorithms with $N=50$ and varying $M$ from $10 \%$ to $100 \%$ of $N$ ( 5 to 50$)$. $D_{m}$ remains constant when resourcesharing is not possible due to the violation of QoS requirements as illustrated at $M=10$ and $M=20$ for all four algorithms. $D_{m}$ increases as more valid pairings are established. Using DA or random allocation algorithms result in a reduced performance. For the DA algorithm, $\forall c_{i} \in H_{c}$, will have $\mu\left(c_{i}\right)=\varnothing$, resulting in a reduction of $D_{m}$ while the random approach does not consider any optimisation objectives in the matching process and hence also results in a reduced performance. The p-DA algorithm performance is as good as the centralised algorithm because the length of the preference list is considered and priority is given to CUEs with smaller preference lists at every round of the iteration process. The $\mathrm{p}$ DA algorithm achieves $12 \%$ to $14 \%$ increase in $D_{m}$ for $M>$ 30 in comparison to the DA and random algorithms. As more
TABLE I. MAIN SIMULATION PARAMETERS

\begin{tabular}{|c|c|}
\hline Parameter & Value \\
\hline Carrier frequency, $f_{c}$ & $2 \mathrm{GHz}$ \\
\hline System bandwidth & $10 \mathrm{MHz}$ \\
\hline Number of resource blocks $(\mathrm{RB}), K$ & 50 \\
\hline RB bandwidth & $180 \mathrm{kHz}$ \\
\hline Maximum CUE transmit power, $\mathrm{P}_{c_{i}, \text { max }}$ & $23 \mathrm{dBm}$ \\
\hline Maximum DUE transmit power, $\mathrm{P}_{d_{j}, \text { max }}$ & $23 \mathrm{dBm}$ \\
\hline D2D distance, $L_{d T, d R}$ & $10 \mathrm{~m} \leq L_{d T, d R} \leq 20 \mathrm{~m}$ \\
\hline CUE SINR Threshold, $\Gamma_{c_{i}, \min }$ & $7 \mathrm{~dB}$ \\
\hline DUE SINR Threshold, $\Gamma_{d_{j}, \text { min }}$ & $3 \mathrm{~dB}$ \\
\hline Noise power density & $-174 \mathrm{dBm} / \mathrm{Hz}$ \\
\hline Number of CUEs, $N$ & 50 \\
\hline Number of DUEs, $M$ & 50 \\
\hline Reliability for DUE, $p_{R_{0}}$ & $10^{-5}$ \\
\hline
\end{tabular}

TABLE II. CHANNEL MODEL FOR LINKS [25], [26], [27]

\begin{tabular}{|c|c|c|c|}
\hline Parameter & $\begin{array}{l}\text { In-factory } \\
\text { DUE link }\end{array}$ & UE-UE link & BS-UE link \\
\hline $\begin{array}{l}\text { Path loss } \\
\text { model }\end{array}$ & $\begin{array}{l}36.8 \log _{10}(d[\mathrm{~km}]) \\
+94.2 \\
+20 \log _{10} \frac{f_{c}(\mathrm{MHz})}{5}\end{array}$ & $\begin{array}{l}40 \log _{10}(d[\mathrm{~km}]) \\
+49 \\
+30 \log _{10} f_{c}(\mathrm{MHz})\end{array}$ & $\begin{array}{l}37.6 \log _{10}(d[\mathrm{~km}]) \\
+58.83 \\
+21 \log _{10} f_{c}(\mathrm{MHz})\end{array}$ \\
\hline Shadowing & $4 \mathrm{~dB}$ & $6 \mathrm{~dB}$ & $8 \mathrm{~dB}$ \\
\hline Fast fading & Rayleigh Fading & Rayleigh Fading & Rayleigh Fading \\
\hline
\end{tabular}

DUEs, $M$, are introduced to the system, when $D_{m}=N$, the network reaches saturation and no DUE will be accessed.

In Fig. 4, we show the total DUE throughput with respect to $M$. Random allocation achieves the worst performance as expected followed by the DA algorithm. The performance of $\mathrm{p}$ $\mathrm{DA}$ is comparable to the centralised approach. The p-DA algorithm achieves $13.57 \%$ and $28.02 \%$ higher throughput compared to DA and random approaches respectively. The centralised approach achieves $15.74 \%$ and $29.8 \%$ higher DUE throughput compared to DA and random algorithm respectively at $M>35$.

In Fig. 5, it is shown that the overall system throughput performance increases with $M$. The centralised approach is comparable to the DA scheme and outperforms the random approach as expected. This is mainly because the matching is based on CUE-DUE assignments that provide higher system throughput. The performance of p-DA and DA algorithms are close and significant difference are apparent as $M>35$, where the p-DA scheme shows an improved system throughput compared to the centralised optimisation scheme. The random approach has the worst performance as expected since no preference or utility maximisation is considered once the QoS criteria is satisfied.

In Fig. 6. we demonstrate the effects of increasing $M$ on the quality of the CUE links. There is a reduction in the total CUE throughput as $M$ increases as expected. Since CUEs have preallocated sub-channels, the introduction of DUEs will cause the total CUE throughput to decrease as a result of the rate loss caused by interference generated by the DUEs as $D_{m}$ increases. This explains the lower performance of the centralised and the p-DA approaches compared to the random and DA approaches as $M \geq 35$.

The performance of the four approaches is compared by investigating the effects of varying the maximum transmit power, $\mathrm{P}_{\max }$ on the number of admitted DUEs, $D_{m}$. It is concluded that $D_{m}$ increases with $\mathrm{P}_{\max }$ for all approaches as shown in Fig. 7. This is as a result of the increase in the $\left|R_{c_{i}}^{d}\right|$ and $\left|R_{d_{j}}^{c}\right|$ for certain CUEs and DUEs respectively. This translates to an increase in the number of eligible CUEs, $n$ and 


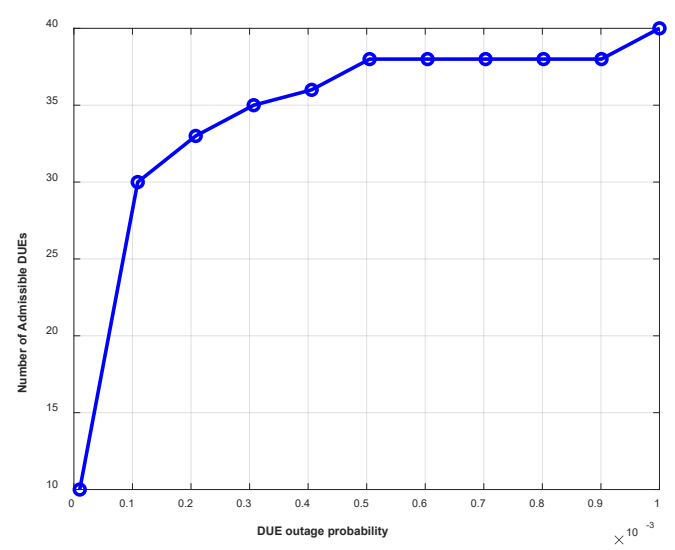

Fig. 2. The number of admissible DUEs, $n$ varying the outage probability, $p_{R_{0}}$, where $M=N=50$.

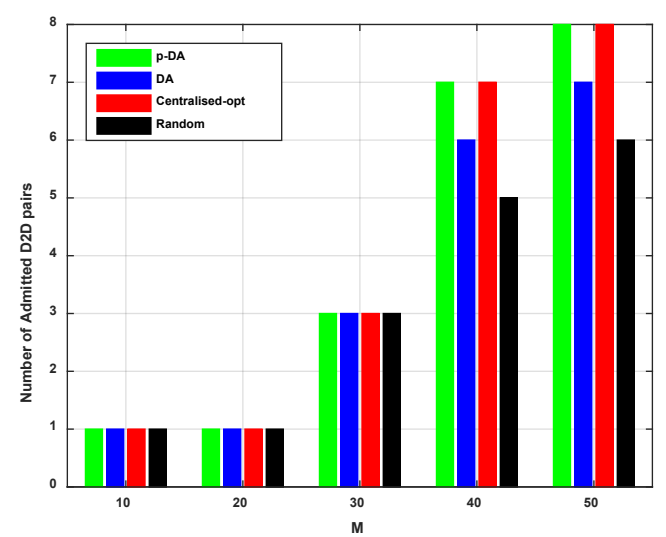

Fig. 3. The number of admitted DUEs, $D_{m}$ varying the number of DUEs, $M$ in the network where $N=50, \mathrm{P}_{c_{i} \max }=23 \mathrm{dBm}$

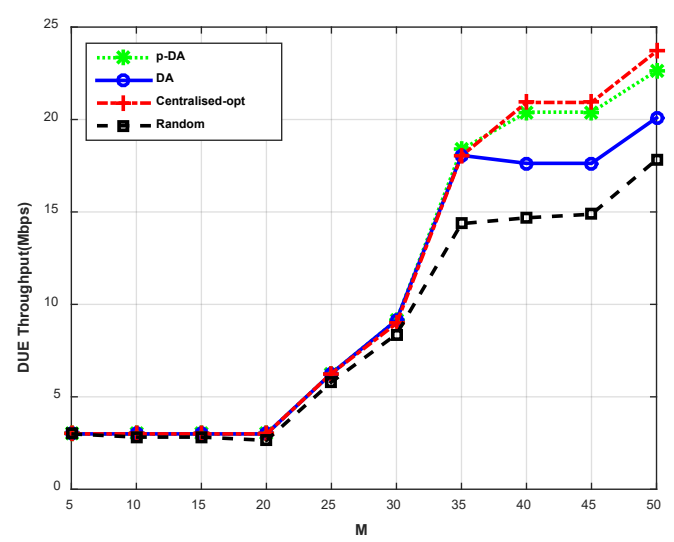

Fig. 4. The total DUE throughput with different number of DUEs, $M$ in the network where $N=50, \mathrm{P}_{c_{i}} \max =23 \mathrm{dBm}$

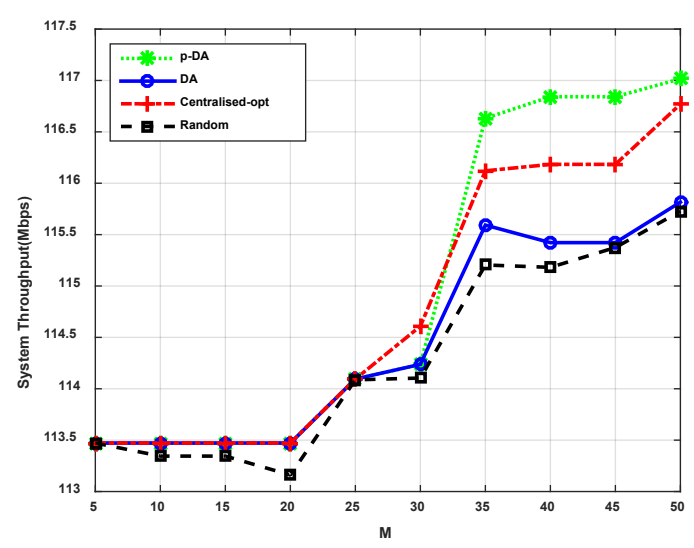

Fig. 5. System throughput with different number of DUEs, $M$ in the network where $N=50, \mathrm{P}_{c_{i} \text { max }}=23 \mathrm{dBm}$

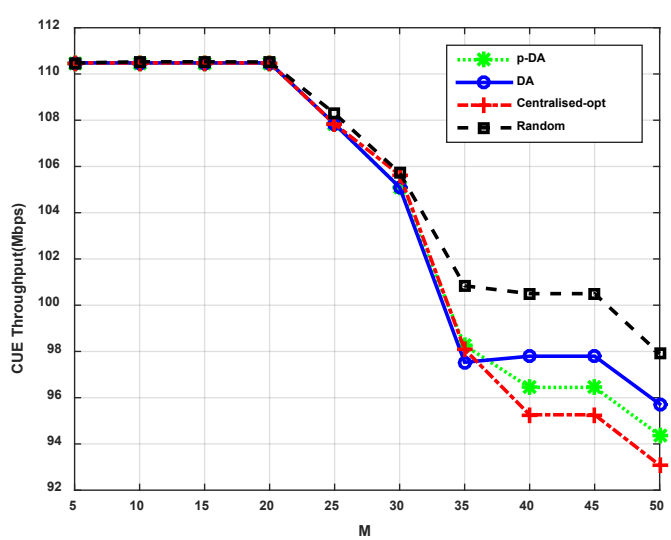

Fig. 6. Total CUE throughput with different number of DUEs, $M$ in the network where $N=50, \mathrm{P}_{c_{i} \max }=23 \mathrm{dBm}$

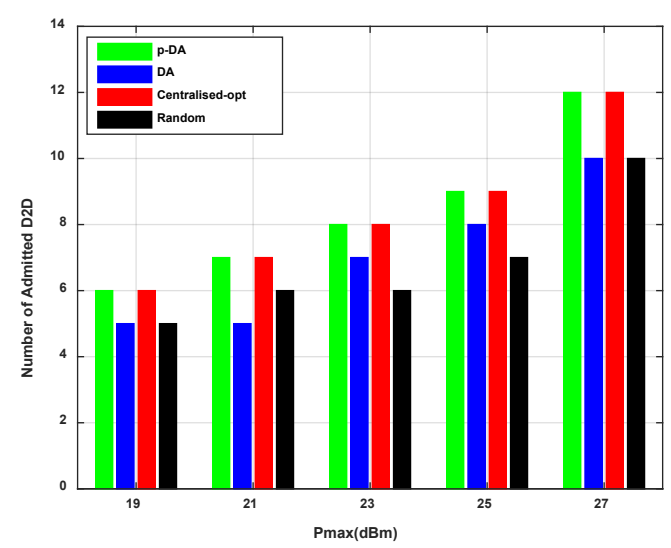

Fig. 7. The number of admitted DUEs, $D_{m}$ versus maximum transmit power and $N=M=50$

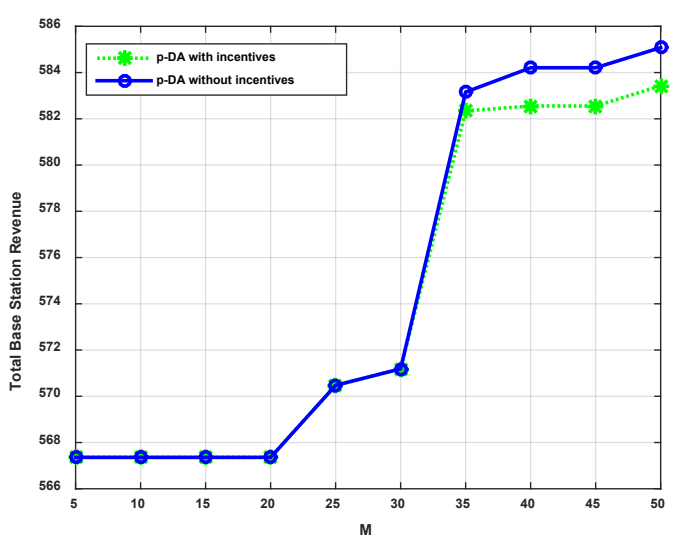

Fig. 8(a). Total base station revenue with different number of DUEs, $M, \mathrm{P}_{c_{i}, \max }=23 \mathrm{dBm}, N=50, \psi=5 \times 10^{-6}$ for the p-DA algorithm.

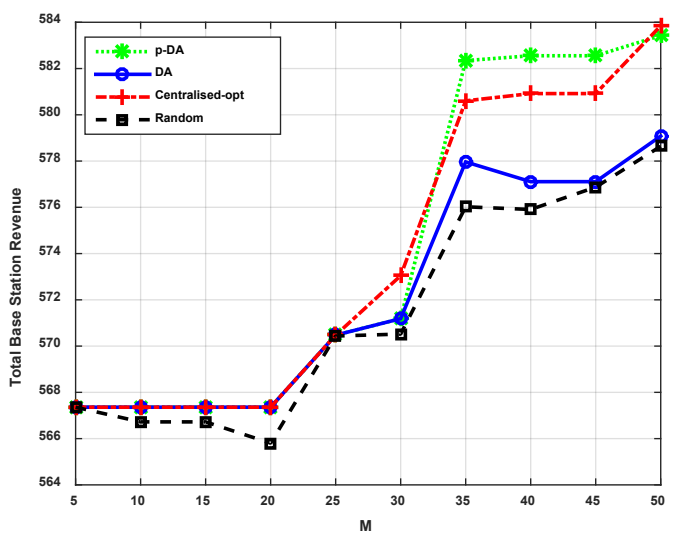

Fig. 8(b). Total base station revenue for different number of DUEs, $M, \mathrm{P}_{c_{i}, \max }=23 \mathrm{dBm}, N=50, \psi=5 \times 10^{-6}$ 
admissible DUEs, $m$, therefore increasing the potential of CUEDUE pairings. It is observed that the optimal power allocation, $\left(P_{c_{i}}^{*}, P_{d_{j}}^{*}\right)$, for a pairing $\left(c_{i}, d_{j}\right)$ is increased by the same factor ( $2 \mathrm{dBm}$, for the studied scenario) as $\mathrm{P}_{\max }$ increases (i.e. from $19 \mathrm{dBm}$ to $21 \mathrm{dBm}, 21 \mathrm{dBm}$ to $23 \mathrm{dBm}$ and so on). Again, random matching achieves the worst performance followed by the DA algorithm. The p-DA algorithm is as the good as the centralised optimisation approach.

We then compare the total revenue generated for the four algorithms. First, we investigate the impact of the rate loss price on the total revenue generated for p-DA, by comparing $U_{B}^{*}(\pi)$ and $U_{B}(\pi)$ for different number of DUE, $D_{m}$ in Fig. 8(a). We observe that rate loss price, $\pi_{\mathcal{L}}$, used to incentivise DUEs $d_{j} \in$ $D_{-}$has a little effect on $U_{B}^{*}(\pi)$ because it is compensated for rate price increase (or gain) by DUEs $d_{j} \in D_{+}$. In particular, at $M \leq 30, U_{B}^{*}(\pi)=U_{B}(\pi)$ because the DUEs belong to $d_{j} \in$ $D_{D A}$, thus no incentives were given. At $M>30$, it is seen that $U_{B}^{*}(\pi)$ is only $0.13 \%$ to $0.28 \%$ less than $U_{B}(\pi)$ which is a good tradeoff to guarantee stability, improved DUE access rate and throughput. In Fig. 8(b), it is seen that the revenue is commensurate with the achieved system throughput (i.e. comparing with Fig. 5) for all four algorithms. The p-DA (with incentives) and centralised algorithms are comparable (which further shows that $\pi_{\mathcal{L}}$ did not significantly affect the total BS revenue) and achieve higher revenues compared to the DA and random algorithms.

\section{CONCLUSIONS}

We investigated and compared the performances of different radio resource management approaches (centralised and distributed) for a D2D-enabled cellular network targeting wireless industrial applications in factories of the future. The aim is to maximise the overall system throughput comprising URLLC D2D links and cellular users with strict QoS requirements. We presented a priced-deferred acceptance ( $p$ DA) algorithm which uses an incentive-based stability to optimise spectrum sharing. Simulation results show that the pDA distributed algorithm is as good as the centralised optimisation approach in terms of DUE access rate and achieved throughput, with the additional advantage of a muchreduced signalling overhead. In comparison with the classical DA algorithm and the random matching method, the p-DA approach shows improvement in terms of the number of admitted DUEs and DUE throughput.

This work focuses on uplink interference and limited to resource-sharing for single CUE-DUE pair. The future work will consider downlink interference scenarios and the reuse of CUE resource blocks by multiple DUEs. We also aim at investigating and comparing the above algorithms with machine learning approaches.

\section{REFERENCES}

[1] 3GPP TR 138 913, "5G: Study on scenario and requirements for next-generation access technologies (Release 14).

[2] B. Holfeld et al., "Wireless communication for factory automation: an opportunity for LTE and 5G systems," IEEE Communications Magazine, vol. 55, no. 6, pp. 36-43, June 2016.

[3] B. Chen, J. Wan, L. Shu, M. Mukherjee and B. Yin, "Smart factory of industry 4.0: key technologies, application case and challenges," IEEE Access, vol. 6, pp. 6505-6519, Dec. 2017.

[4] O.N. Yilmaz, Y.P. Wang, N.A Johansson, N. Brahmi, S.A. Ashraf and J. Sachs, "Analysis of ultra-reliable and low-latency $5 \mathrm{G}$ communication for a factory automation use case," in Proc. IEEE international conference on communication workshop (ICCW), pp. 1190-1195, June 2015.

[5] H. Chen et al., "Ultra-reliable low latency cellular networks: use cases, challenges and approaches," IEEE Communications Magazine, vol. 56, no. 12, pp. 119-125, Sep. 24, 2018.
J. Ansaria et al., "Demo: a realistic use-case for wireless industrial automation and control," in Proc. IEEE International Conference on Networked Systems (NetSys), pp. 1-2, Mar. 2017.

[7] J. Wan et al., "Toward dynamic resources management for IoTbased manufacturing," IEEE Communications Magazine, vol. 56, no. 2, pp. 52-59, Feb 2018

[8] A. Asadi, Q. Wang and V. Manuso, "A survey on device-to-device communication in cellular networks," IEEE Commuincations Surveys and Tutorials, vol. 16, no. 4, pp. 1801-1819, Apr. 2014.

[9] R. H. Tehrani, S. Vahid, D. Triantafyllopoulou, H. Lee and K. Moessner, "Licensed spectrum sharing schemes for mobile operators: a survey and outlook," IEEE Communications Surveys \& Tutorials, vol. 18, no.4, Fourth Quarter 2016.

[10] W. Sun, E. G. Ström, F. Brännström, K. Cheong Sou, and Y. Sui, "Radio resource management for D2D-based V2V communication," IEEE Transactions on Vehicular Technology, vol. 65, no. 8 pp. 6636-6650, 2015.

[11] L. Liang, G.Y. Li and W. Xu, "Resource allocation for D2D-enabled vehicular communications," IEEE Transactions on Communications, vol. 65, no. 7, pp. 3186-3197, Jul 2017.

[12] G. Yu, L. Xu, D. Feng, R. Yin, G. Ye Li, and Y. Jiang. "Joint mode selection and resource allocation for device-to-device communications," IEEE Transactions on Communications, vol. 62, no. $11,3814-3824,2014$

[13] Z. Han et al., "Game theory in wireless and communication networks: theory, models and applications" Cambridge University Press, Oct. 2011.

[14] Y. Gu, W. Saad, M. Bennis, M. Debbah, and Z. Han, "Matching theory for future wireless networks: fundamental and applications," IEEE Communication Magazine, vol. 53, no. 5, pp 52-59, May 2015.

[15] D. Gale and L. Shapely, "College admission and stability of marriage," The American Mathematical Monthly, vol. 69, no.1, pp.9-15, Jan. 1962.

[16] Y. Gu, Y. Zhang, M. Pan, and Z. Han, "Cheating in matching of device to device pairs in cellular networks," in Proc. IEEE Global Communications Conference (GLOBECOM), Austin, TX, Dec. 2014.

[17] M. T. Islam, A. M. Taha, S. Akl and M. Abu-Elkheir, "A stable algorithm for resource allocation for underlaynig device-to-device communications", in Proc. IEEE International Conference on Communication (ICC), Malaysia, May 2016.

[18] X. Mao, B. Zhang, Y. Chen, J. Yu and Z. Han, "Matching game based resource allocation for $5 \mathrm{G} \mathrm{H}$-CRAN networks with device-todevice communication," in Proc. IEEE Personal Indoor and Mobile Radio Communication (PIMRC), Montreal Canada, Oct. 2017.

[19] S. M. Ahsan Kazmi, N. H. Tran, T. M. Ho, D. K. Lee and C. S. Hong, "Decentralised spectrum allocation in D2D underlying cellular networks," in Proc. IEEE Asia-Pacific Network Operations and Management Symposium (APNOMS), Kanasawa, Japan, Oct. 2016.

[20] Y. Wang and L. Wang, "Matching theory-based cooperative secure transmission strategy for social-aware D2D communications,"IEEE Transactions on Vehicular Technology, vol. 68, no. 10, pp. 1028910294, Aug 52019.

[21] B. Wang, Y. Sun, H.M. Nguyen and T.Q. Duong, "A novel socially stable matching model for secure relay selection inD2D communications," IEEE Wireless Communications Letters, vol. 9, no. 2, pp. 162-165, Oct 112019.

[22] M. Rasti, A. R. Sharafat, and J. Zander, "A distributed dynamic target-SIR-tracking power control algorithm for wireless cellular networks," IEEE Transactions on Vehicular Technology, vol. 59, no. 2, pp. 906-916, 2009.

[23] H. Wang, and X. Chu, "Distance-constrained resource-sharing criteria for device-to-device communications underlaying cellular networks," Electronics letters, vol. 48, no. 9, pp. 528-530, 2012.

[24] Y. Xiao, K. Chen, C. Yuen and L. A. DaSilva, "Spectrum sharing device-to-device communications in cellular networks: a game theoretic approach," in Proc. IEEE International Symposium on Dynamic Spectrum Access Networks (DYSAN), McLean, VA, USA, Apr. 2014.

[25] WINNER II Channel Models, Standard IST-4-027756 WINNER II D1.1.2 V1.2, Sep. 2007.

[26] H. Xing and S. Hakola, "The investigation of power control schemes for a device-to-device communication integrated into ofdma cellular system," in Proc. IEEE Personal Indoor and Mobile Radio Communication (PIMRC), pp. 1775-1780, Sep. 2010.

[27] Evolved Universal Terrestrial radio Access (E-UTRA), "Further Advancements for E-UTRA Physical Layer Aspects (Release 9)," 3GPP TR 36.814, Tech. Rep., 2010. 